\section{Antiviral Therapy during the Coronavirus Disease (COVID-19) Pandemic: Is It Appropriate to Treat Patients in the Absence of Significant Evidence?}

\section{THE "PRO" SIDE}

The strengths of formal research to evaluate investigational therapies are well known. In an ideal world, when a new question or problem is recognized, rigorous testing in well-designed clinical trials would be performed. However, the coronavirus disease (COVID-19) pandemic has given new meaning to the word "unprecedented". Here, we argue that it is ethically appropriate to offer investigational agents outside of a clinical trial during an emergency, such as the current COVID-19 pandemic, and that the precedent to do so has been set in other outbreaks, such as the Ebola epidemics in Africa. The World Health Organization (WHO) has developed an ethical framework for the use of investigational agents, called MEURI, which stands for "monitored emergency use of unregistered and investigational interventions". ${ }^{1}$ According to MEURI, the criteria for using investigational agents outside of clinical trials are the following: no proven effective therapy exists; it is not possible to initiate clinical trials immediately; data providing preliminary support for the investigational agents' safety and efficacy exist (i.e., in vitro or animal studies and support from clinical experts); relevant country authorities and ethics committees have approved such use; adequate resources are in place to minimize risk; the patient's informed consent has been obtained; and the use is monitored, with the results being documented and shared with the scientific community in a timely fashion. The WHO has stated that no proven therapy exists for the disease caused by the severe acute respiratory syndrome coronavirus 2 (SARS-CoV-2), and we argue that the other criteria for MEURI are met as well.

\section{Argument 1: Research Bureaucracy Is Inefficient and Prohibitive-Lessons from Ebola}

In March 2014, an outbreak of Ebola was declared in Guinea, but it was not until 5 months later, in August 2014, that the WHO declared a public health emergency of international concern. A research group developed a protocol for clinical investigation of brincidofovir, a process that took 3 months, and another 6 weeks was consumed by additional bureaucratic delay. The narrative account, published in Nature in 2015, described the pace at which the trial was started as "unprecedented" (in its rapidity), in contrast to the norm of 18 months, ${ }^{2}$ yet during that time the epidemic hit its peak, ${ }^{3}$ and affected patients had no access to potentially life-saving investigational agents through clinical trials. The author's advice for the future was the following: "Government leaders must give the
WHO the money and support it needs to ensure that the world is 'research ready' for the next outbreak. A properly funded and empowered WHO could oversee the design and implementation of an on-call global task force of clinical-trial staff."

Perhaps this would be a viable strategy for a limited outbreak of a predicted pathogen; however, COVID-19 demonstrates that in a truly global outbreak, such a strategy would be grossly insufficient. We applaud the research response to COVID-19 thus far. At the time of writing, on May 14, 2020, an incredible 1486 protocols had been registered at www.clinicaltrials.gov, and many clinical trials have been initiated by the $\mathrm{WHO}$ and other investigators within extremely short timeframes. At the same time, however, there were more than 4.2 million confirmed cases worldwide, or about 2826 cases per clinical protocol. Of course, not all cases would be appropriate for drug therapy, but it is inconceivable that research protocols could be mobilized to enrol even a fraction of those who are eligible.

\section{Argument 2: Variation in Research Infrastructure and Capacity May Introduce Inequities}

Equity and fairness are foundational ethical principles in the management of outbreak resources. ${ }^{4,5}$ Even if rapidly mobilized, clinical research is generally associated with urban universities. Residents of smaller centres therefore have less opportunity to access investigational therapy through clinical trials, which represents a substantial inequity in the availability of potentially life-saving therapy. In addition, clinical trials often exclude those with a lower likelihood of response or deemed to be at higher risk, such as pregnant or elderly patients. ${ }^{6-8}$ The WHO's MEURI emphasizes the ethical principle of patient autonomy or the right of patients to make their own risk/ benefit assessments in accordance with their own personal values, goals, and health conditions. Excluding patients because of geography or demographic characteristics that would not exclude them from on-label drug prescriptions overrides this principle of patient autonomy.

On March 24, 2020, the British Columbia Centre for Disease Control issued a statement indicating a preference for clinical trials, with the additional proviso that where such trials are not available, compassionate use of therapies is appropriate, provided patients are advised of the risks and benefits and safety data are collected. ${ }^{9}$ These directions align with the WHO's guidance on MEURI and with the explanation of Gostin and Berkman, in the second of the WHO discussion papers addressing ethical issues in pandemic influenza planning. ${ }^{10}$ In that document, the authors recognize the provision of investigational agents as ethically appropriate, provided that they are "proportionate in terms of benefits and burdens" and that resources for population-based research are inadequate.

Canada's health care system may not be designed to allow equitable access to new therapies through clinical trials in the context 
of widespread COVID-19 infection, but it is able to support access to investigational therapies in a safe and monitored environment per the conditions of MEURI.

\section{Argument 3: Absence of Evidence Is Not Evidence of Absence (of Positive or Negative Effects)}

A common logical fallacy suggests that an untested therapy has no effect. This is a non sequitur. If the therapy is untested, the only reasonable conclusion is that its efficacy is unknown. However, the situation is rarely as simple as a complete absence of study data. Clinicians are often faced with the dilemma of poor evidence or data from one condition that they are attempting to extrapolate to another. In the absence of strong evidence, therapies are routinely prescribed that are considered unproven. Indeed, some therapies informed by low-quality evidence are commonly recommended, including aminoglycosides or ceftriaxone in enterococcal endocarditis, ${ }^{11}$ combination antibiotics for persistent methicillin-resistant Staphylococcus aureus bacteremia, ${ }^{12}$ and almost every off-label indication for any therapy. ${ }^{13}$ Investigational agents for COVID-19 may not have proven efficacy, but equally they are not proven to be inefficacious. For all of the agents currently proposed for the treatment of COVID-19, biological plausibility for activity has been demonstrated and human safety data are available, therefore meeting the WHO MEURI criteria for a scientific rationale and support of clinical experts in the field.

\section{Conclusion}

The modern world has no experience with a pandemic of this proportion. The medical and scientific community has much to learn about this virus and the associated disease. Formal acquisition of knowledge through clinical trials is highly desirable, but it accumulates at a far slower pace than the pandemic has progressed to date. The ideal for evaluation of therapy in clinical trials must be balanced against efficiency, equity, autonomy, and beneficence. We do not advocate for indiscriminate use, but we do advocate for access to reasonably safe and possibly effective therapy under clinician oversight and with informed patient consent, as suggested by guidance documents of the WHO.

\section{References}

1. Guidance for managing ethical issues in infectious disease outbreaks. World Health Organization; 2016.

2. Lang T. Ebola: embed research in outbreak response. Nature. 2015; 524(7563):29-31.

3. 2014 ebola outbreak in West Africa epidemic curves. Centers for Disease Control and Prevention (US); 2019 Apr 3 [cited 2020 May 14]. Available from: https:/www.cdc.gov/vhf/ebola/history/2014-2016-outbreak/cumulativecases-graphs.html

4. Verweij M. Equitable access to therapeutic and prophylactic measures. In: Addressing ethical issues in pandemic influenza planning: discussion papers. WHO/HSE/EPR/GIP/2008.2; WHO/IER/ETH/2008.1. World Health Organization; 2008. p. 1-28.

5. Ethical considerations in developing a public health response to pandemic influenza. WHO/CDS/EPR/GIP/2007.2. World Health Organization; 2007.

6. Van der Graaf R, Van der Zande ISE, Den Ruijter HM, Oudijk MA, Van Delden JJM, OudeRengerink K, et al. Fair inclusion of pregnant women in clinical trials: an integrated scientific and ethical approach. Trials. 2018;19(1):Article 78
7. van der Graaf R, van der Zande ISE, van Delden JJM. How the CIOMS guidelines contribute to fair inclusion of pregnant women in research. Bioethics. 2019;33(3):367-73.

8. Shemoy P, Harugeri A. Elderly patients' participation in clinical trials. Perspect Clin Res. 2014;6(4):184-9.

9. Recommendation: therapies for COVID-19. In: Treatments: clinical guidance. BC Centre for Disease Control; 2020 Mar 24 [updated 2020 May 11; cited 2020 May 14]. Available from: http://www.bccdc.ca/health-professionals/ clinical-resources/covid-19-care/clinical-care/treatments

10. Gostin L, Berkman B. Isolation, quarantine, border control and socialdistancing measures. In: Addressing ethical issues in pandemic influenza planning: discussion papers. WHO/HSE/EPR/GIP/2008.2; WHO/IER/ ETH/2008.1. World Health Organization; 2008. p. 29-65.

11. Baddour LM, Wilson WR, Bayer AS, Fowler VG Jr, Tleyjeh IM, Rybak MJ, et al. Infective endocarditis in adults: diagnosis, antimicrobial therapy, and management of complications: a scientific statement for healthcare professionals from the American Heart Association. Circulation. 2015; 132(15):1435-86.

12. Liu C, Bayer A, Cosgrove SE, Daum RS, Fridkin SK, Gorwitz RJ, et al. Clinical practice guidelines by the Infectious Diseases Society of America for the treatment of methicillin-resistant Staphylococcus aureus infections in adults and children. Clin Infect Dis. 2011;52(3):e18-55.

13. Wittich CM, Burkle CM, Lanier WL. Ten common questions (and their answers) about off-label drug use. Mayo Clin Proc. 2012;87(10):982-90.

Bruce R Dalton, BScPharm, PharmD

Pharmacy Clinical Practice Leader and Infectious Diseases Pharmacist Department of Pharmacy Services

Alberta Health Services

Calgary, Alberta

Linda D Dresser, BSCPharm, PharmD, FCSHP

Pharmacotherapy Specialist, Antimicrobial Stewardship University Health Network

Assistant Professor, Leslie Dan Faculty of Pharmacy

University of Toronto

Toronto, Ontario

Competing interests: Linda Dresser has received personal fees from Sunovion for an educational event outside the scope of this article. No other competing interests were declared.

\section{THE "CON" SIDE}

\section{The Issue of Using Unproven Treatments}

The emergence and rapid transmission of the coronavirus disease (COVID-19), with its relatively high risk of death among patients with comorbidities and severe disease, are compelling the medical community to make decisions before the underlying science has been fully developed. Because there are no licensed vaccines or drug treatments for COVID-19, clinicians have been forced to consider investigational and unproven treatment alternatives in the absence of solid scientific data. When clinicians are confronted with severely ill patients who are at risk of dying, it seems obvious that they should immediately attempt to treat patients with "something", even if the medications available are unproven. However, such action is associated with major pitfalls, 
which include placing patients at substantial risk of harm (from adverse events) that outweighs the benefits, wasting precious resources in times of urgency, compromising the conduct of clinical trials, and offering false hope to patients and their family members. When a clinician faces a dilemma in which limited data are available to support treatment, what should they do? We believe that the appropriate scientific and ethical approach is for experimental and unproven medications-including treatments considered for "emergency use" and "compassionate use" - to be made accessible to patients only through clinical trials.

\section{The Concept of Evidence-Based Medicine}

The World Health Organization (WHO), in its interim guidance on clinical management of severe acute respiratory infection in patients with suspected COVID-19, states that "Use of investigational anti-COVID-19 therapeutics should be done under ethically approved, randomized, controlled trials."

Evidence-based medicine, which requires that treatments be based on established scientific evidence, is the current paradigm for the practice of medicine. ${ }^{2}$ Before a drug can be licensed for commercial use in humans, it must undergo a rigorous scientific development process involving clinical trials. The main purpose of this methodological approach is to determine, to the best of the researchers' ability, the safety and efficacy of the drug for its intended indications; the data generated in this way form the basis of evidence-based practice. This process, however, does not ensure the safety or efficacy of the medication when used for nonintended indications, and patients may be placed at risk for adverse events in those circumstances. For example, during the outbreak of severe acute respiratory syndrome in 2003, ribavirin (an antiviral agent) was used experimentally, despite a lack of clinical trial data, but was later found to be ineffective and harmful to patients, causing hemolytic anemia, liver dysfunction, and poor outcomes. ${ }^{3}$ This misuse of ribavirin highlights the importance of properly conducted, robust clinical studies to assess the effects and safety of drug therapy.

\section{The Perils of Emotionally Based Medicine}

On March 19, 2020, US President Donald Trump, in his White House briefing, stated that hydroxychloroquine "showed tremendous promise" and "could be a game-changer." "This statement has caused numerous clinicians and patients to consider using hydroxychloroquine even though data regarding its safety and efficacy are limited.

In fact, the evidence for hydroxychloroquine as a treatment for COVID-19 is still evolving. At the time of writing (mid-May 2020), this drug has been shown to exhibit in vitro antiviral activity against the severe acute respiratory syndrome coronavirus 2 (SARS-CoV-2), ${ }^{5}$ and preliminary clinical reports have shown both positive and negative results. ${ }^{6-8}$ Clinicians are trained to "do no harm", so they feel an ethical obligation to offer treatment; however, simply acting on this feeling and starting hydroxychloroquine for all patients with COVID-19 would represent a great leap of faith. Hydroxychloroquine and a similar drug, chloroquine, failed in clinical trials, despite having in vitro activity against several viruses, including the Ebola, influenza, HIV, dengue, and Chikungunya viruses. Even worse, chloroquine has been shown to cause harm in an animal model for Chikungunya. ${ }^{9}$ Interventions may not offer any more value than best estimated chance. In COVID-19 studies, the dosing regimen for hydroxychloroquine varies considerably, and the occurrence of serious cardiac arrhythmias has been described. ${ }^{8}$ In addition, patients treated with unproven therapy may be given false hope, which adds a psychological component to the complexities when determining benefits of treatment. ${ }^{10}$ Furthermore, any data collected from such patients would not be reliable when making informed health care decisions for evidence-based practice changes. ${ }^{11}$ Thus, unproven interventions require thorough study and should not be offered outside of clinical trials.

\section{The Effects on the Community}

In a disaster response, the widespread use of unproven interventions — for both "emergency use" and "compassionate use" with inadequate collection of data concerning patient outcomes must be avoided. ${ }^{12}$ The use of unproven treatments on humans without proper oversight can lead to devastating consequences. Unscrutinized prescribing and dispensing of medications can distort access to therapy for patients who would otherwise benefit from these treatments. ${ }^{13}$ In the case of hydroxychloroquine, which is usually prescribed for the management of lupus and arthritis (among other diseases), indiscriminate use for prevention or treatment of COVID-19 may lead to drug shortages, reducing access to the drug by patients who have those other conditions. ${ }^{4}$ Such shortages can increase anxiety and uncertainty for all patients. In addition, the availability of investigational and unproven treatments outside of clinical trials would interfere with the ability to conduct research and thus would subsequently reinforce knowledge deficits. ${ }^{10}$ Enrolment of patients into clinical trials may be affected, because some patients would already have access to these medications. There would also be a reduced requirement and urgency for researchers to develop new interventions for management, because the perceived need would be negated..$^{10}$ Furthermore, use of unproven treatments at one site would perpetuate usage at another, as clinicians would seek to emulate the practices of their peers. Ethically, research should be part of the public health response during an epidemic, based on the moral duties of caregivers, as stated by the WHO Working Group on Ethics and COVID-19. ${ }^{14}$

\section{The Overall Approach}

We believe that evidence-informed clinical studies should be the foundation of any treatment used for COVID-19. To deviate from this approach and "jump the gun" on the science would place patients at risk of adverse events, poor outcomes, psychological stresses, reduced medication access, uncertainty and wastage of valuable resources, and would cause confusion for clinicians. 
In summary, we feel that the statement from the British Columbia Centre for Disease Control (as quoted in a joint statement of the College of Physicians and Surgeons of British Columbia, the College of Pharmacists of British Columbia, and the British Columbia College of Nurse Professionals ${ }^{15}$ ) effectively summarizes our opinions:

It is important to understand that there are potential harms to the patient, risks to our understanding of what is truly a beneficial treatment or not, and depleting access to therapies known to be helpful or essential in other disease states. For these reasons, the use of unproven therapies for COVID 19 is not recommended outside clinical trials.

\section{References}

1. Clinical management of severe acute respiratory infection when COVID19 disease is suspected [interim guidance]. World Health Organization; 2020 Mar 13 [cited 2020 May 18]. Available from: https://www.who.int/ publications-detail/clinical-management-of-severe-acute-respiratoryinfection-when-novel-coronavirus-(ncov)-infection-is-suspected

2. Landry JT, Foreman T, Kekewich M. Reconsidering the ethical permissibility of the use of unregistered interventions against Ebola virus disease. Camb Q Healthc Ethics. 2015;24(3):366-9.

3. Stockman LJ, Bellamy R, Garner P. SARS: systematic review of treatment effects. PLoS Med. 2006;3(9):e343.

4. Peschken CA. Possible consequences of a shortage of hydroxychloroquine for lupus patients amid the COVID-19 pandemic. J Rheumatol. 2020 Apr 8; pii: jrheum.200395. doi: 10.3899/jrheum.200395 [Epub ahead of print].

5. Yao X, Ye F, Zhang M, Cui C, Huang B, Niu P, et al. In vitro antiviral activity and projection of optimized dosing design of hydroxychloroquine for the treatment of severe acute respiratory syndrome coronavirus 2 (SARS-CoV-2). Clin Infect Dis. 2020 Mar 9; pii: ciaa237. doi: 10.1093/ $\mathrm{cid} /$ ciaa237 [EPub ahead of print].

6. Gautret P, Lagier JC, Parola P, et al. Hydroxychloroquine and azithromycin as a treatment of COVID-19: results of an open-label nonrandomized clinical trial. Int J Antimicrob Agents. 2020 Mar 20:105949. doi: 10.1016/j.ijantimicag.2020.105949 [Epub ahead of print].

7. Chen J, Liu D, Liu L, Liu P, Xu Q, Xia L, et al. A pilot study of hydroxychloroquine in treatment of patients with common coronavirus disease19 (COVID-19). J Zhejiang Univ (Med Sci). 2020;49(2):215-9.

8. Assessment of evidence for COVID-19-related treatments: updated 5/15/20. Bethesda (MD): American Society of Health-System Pharmacists; 2020 [cited 2020 May 18]. Available from: https:// www.ashp.org/-/media/assets/pharmacy-practice/resource-centers/ Coronavirus/docs/ASHP-COVID-19-Evidence-Table.ashx?la=en\&hash= B414CC64FD64E1AE8CA47AD753BA744EDF4FFB8C\&hash=B414 CC64FD64E1AE8CA47AD753BA744EDF4FFB8C
9. Roques P, Thiberville SD, Dupuis-Maguiraga L, Lum FM, Labadie K, Martinon F, et al. Paradoxical effect of chloroquine treatment in enhancing Chikungunya virus infection. Viruses. 2018;10(5):268.

10. Shah SK, Wendler D, Danis M. Examining the ethics of clinical use of unproven interventions outside of clinical trials during the Ebola epidemic. Am J Bioethics. 2015;15(4):11-6.

11. Ebunoluwa OO, Kareem FA. Ethics of trial drug use: to give or not to give? BEOnline. 2016;3(2):22-40.

12. London AJ. Social value, clinical equipoise, and research in a public health emergency. Bioethics. 2019;33(3):326-34.

13. Kim AHJ, Sparks JA, Liew JW, Putman MS, Berenbaum F, DuarteGarcia A, et al.; COVID-19 Global Rheumatology Alliance. A rush to judgment? Rapid reporting and dissemination of results and its consequences regarding the use of hydroxychloroquine for COVID-19. Ann Intern Med. 2020 Mar 30. doi: 10.7326/M20-1223 [Epub ahead of print]

14. Ethical standards for research during public health emergencies: distilling existing guidance to support COVID-19R\&D. World Health Organization; 2020 Mar 29 [cited 2020 May 18]. Available from: https://www.who.int/ publications-detail/ethical-standards-for-research-during-public-healthemergencies

15. Joint statement on the unproven therapies for COVID-19. Vancouver (BC): College of Physicians and Surgeons of British Columbia; College of Pharmacists of British Columbia; British Columbia College of Nurse Professionals; 2020 Mar 25 [cited 2020 May 18]. Available from: https://www.bcpharmacists.org/readlinks/joint-statement-unproventherapies-covid-19

Daniel J G Thirion, BPharm, MSc, PharmD, FCSHP

Full Clinical Professor, Faculty of Pharmacy

Pharmacist, McGill University Health Centre

Montréal, Quebec

Tim T Y Lau, PharmD, ACPR, FCSHP

Clinical Supervisor and Pharmacotherapeutic Specialist in Infectious Diseases, Pharmaceutical Sciences

Vancouver General Hospital

Clinical Professor, Faculty of Pharmaceutical Sciences, and Associate

Member, Division of Infectious Diseases, Faculty of Medicine

The University of British Columbia

Vancouver, British Columbia

Competing interests: None declared. 